\title{
A facile synthesis of 3-aryl-2-mercaptoacrylic acid under microwave irradiation
}

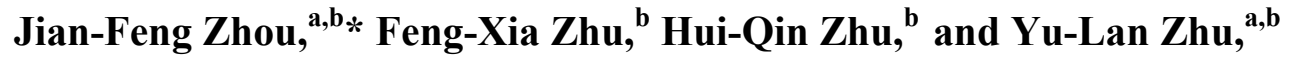 \\ ${ }^{a}$ Department of Chemistry, Huaiyin Teachers College,Huaian 223300, P. R. China \\ ${ }^{b}$ Jiangsu Key Laboratory for Chemistry of Low-Dimensional Materials, \\ Huaian 223300, P. R. China \\ E-mail:jfchou@163.com
}

\begin{abstract}
A series of 3-aryl-2-mercaptoacrylic acid derivatives were synthesized by the hydrolysis of 5-(benzylidene)rhodanines in alkaline solution under microwave irradiation. Compared with the classical reaction conditions, this method has the advantage of excellent yields (75 94\%), short reaction time (3 6 $\mathrm{min}$ ), environmental benign, low energy consumption and easy work-up.
\end{abstract}

Keywords: Rhodanine, benzylidenerhodanines, 3- aryl-2-mercaptoacrylic acids, microwave irradiation

\section{Introduction}

The study of 3-aryl-2-mercaptoacrylic acids as analytical reagents has shown that they act as bidentate ligands and form colored complexes with transition metal ions with great sensitivity and which are easily extractable with organic solvents. ${ }^{1,2}$ The 3-aryl-2-mercaptoacrylic acids can inhibit of neuraminidases. ${ }^{3}$ They were also used as intermediates for the synthesis of a variety of heterocyclic sulphur compounds. ${ }^{4}$ The synthesis of 3-aryl-2-mercaptoacrylic acids derivatives were generally carried out by the hydrolysis of 5-(benzylidene)rhodanines in alkaline solution in refluxing with longer reactions time $(0.5 \sim 1.0 \mathrm{hs}) .{ }^{5,6}$ To the best of our knowledge, although the synthesis of 3-aryl-2-mercaptoacrylic acids derivatives via the hydrolysis of 5-(benzylidene)-

rhodanines in alkaline solution has been studied previously ${ }^{1,3,5,6}$, such a kind of reaction has not been investigated under microwave irradiation until now.

The wide applicability of microwave irradiation in chemical reaction enhancement is due to high reaction rates, formation of cleaner products, and operational simplicity. ${ }^{7,8,9,10}$ Continuing our interest in the development of efficient reagent and simple procedures for the synthesis of organic compounds, we have reported previously a facile synthesis of 5-(arylalkylidene) rhodanines in the presence of tetrabutylammonium bromide as a phase transfer catalyst in water 
under microwave irradiation. ${ }^{11}$ Herein, we would like to report an efficient synthesis of 3-aryl2-mercaptoacrylic acid derivatives which were synthesized by the hydrolysis of 5-(benzylidene)rhodanines in alkaline solution under microwave irradiation (Scheme 1).

The synthetic route is shown as following
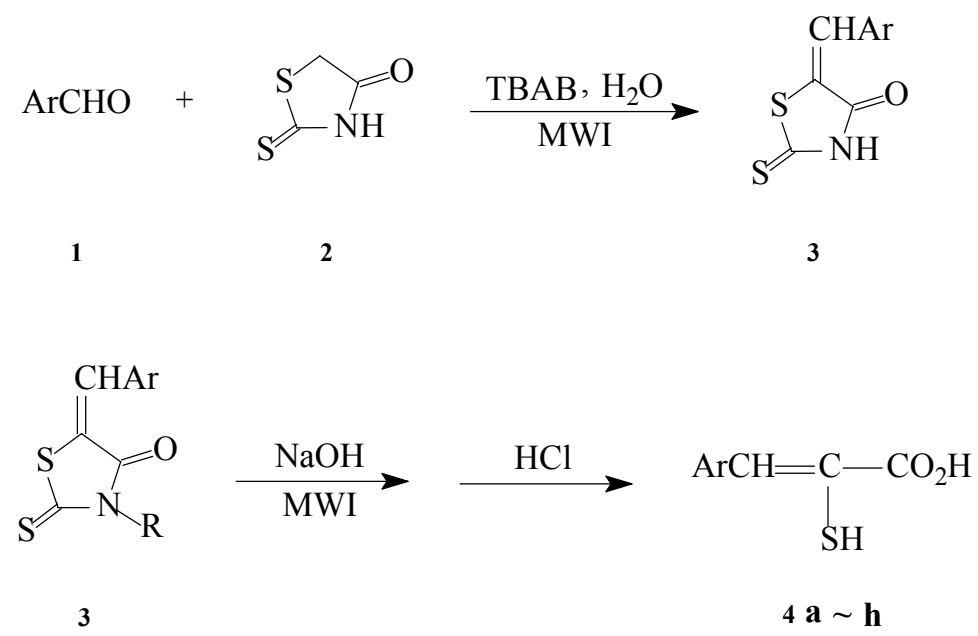

Ar: (a) $\mathrm{C}_{6} \mathrm{H}_{5}$, (b) 4- $\mathrm{CH}_{3} \mathrm{OC}_{6} \mathrm{H}_{4}$, (c) 2-furyl, (d) $\mathrm{C}_{6} \mathrm{H}_{5} \mathrm{CH}=\mathrm{CH}$, (e) 4- $\mathrm{NO}_{2} \mathrm{C}_{6} \mathrm{H}_{4}$, (f) $4-\mathrm{CH}_{3} \mathrm{C}_{6} \mathrm{H}_{4}$, (g) $2-\mathrm{ClC}_{6} \mathrm{H}_{4}$, (h) $4-\mathrm{ClC}_{6} \mathrm{H}_{4}$, (i) $4-\left(\mathrm{CH}_{3}\right)_{2} \mathrm{NC}_{6} \mathrm{H}_{4}$, (j) 3, $4-\left(\mathrm{OCH}_{2} \mathrm{O}\right) \mathrm{C}_{6} \mathrm{H}_{3}$.

\section{Scheme 1}

\section{Results and Discussion}

As is shown in Scheme 1, the hydrolysis of 5-(benzylidene)rhodanines in alkaline solution under microwave irradiation to give 3-aryl-2-mercaptoacrylic acid $4 \mathbf{a}$ in $91 \%$ yield. The results (summarized in Table 1) clearly reveal the scope and generality of the reaction with respect to various substituted aromatic aldehyde, and that reasonable yields (75 94\%) of the products were achieved after only 3 6 min of microwave irradiation. Another important feature of this procedure is the survival of a variety of functional groups, such as ethers, nitro groups, halides, amino, heterocycles etc. under the reaction conditions. All products were characterized by IR, ${ }^{1} \mathrm{H} N \mathrm{NR}$, MS spectral data and Elemental analysis. Their melting points (except $\mathbf{4 e} \sim \mathbf{4 j}$ ) are identical to those of the known compounds reported in the literature. 
Table 1. Synthesis of $\mathbf{4 a} \sim \mathbf{j}$ under microwave irradiation

\begin{tabular}{|c|c|c|c|c|c|}
\hline Compds & $\mathrm{Ar}$ & Time / min & Yields / \%* & $\mathrm{Mp}\left({ }^{\circ}\right)$ & Lit. mp. $\left({ }^{\circ}\right)$ \\
\hline 3a & $\mathrm{C}_{6} \mathrm{H}_{5}$ & 5 & 91 & $135 \sim 136$ & $(133 \sim 134)^{5}$ \\
\hline $3 b$ & $4-\mathrm{CH}_{3} \mathrm{OC}_{6} \mathrm{H}_{4}$ & 6 & 77 & $140 \sim 142$ & $140(\mathrm{dec})^{6}$ \\
\hline $3 c$ & 2 -furyl & 3 & 89 & $115 \sim 116$ & $(115 \sim 117)^{5}$ \\
\hline 3d & $\mathrm{C}_{6} \mathrm{H}_{5} \mathrm{CH}=\mathrm{CH}$ & 5 & 82 & $150 \sim 152$ & $148-151^{5}$ \\
\hline $3 e$ & 4- $\mathrm{NO}_{2} \mathrm{C}_{6} \mathrm{H}_{4}$ & 3 & 94 & $140 \sim 142$ & -- \\
\hline $3 f$ & 4- $\mathrm{CH}_{3} \mathrm{C}_{6} \mathrm{H}_{4}$ & 5 & 78 & $179 \sim 180$ & -- \\
\hline $3 g$ & $2-\mathrm{ClC}_{6} \mathrm{H}_{4}$ & 3 & 80 & $140-143$ & -- \\
\hline $3 \mathrm{~h}$ & $4-\mathrm{ClC}_{6} \mathrm{H}_{4}$ & 3 & 85 & $161-162$ & -- \\
\hline $3 \mathbf{i}$ & 4- $\left(\mathrm{CH}_{3}\right)_{2} \mathrm{NC}_{6} \mathrm{H}_{4}$ & 6 & 75 & $163 \sim 164$ & -- \\
\hline $3 \mathbf{j}$ & $3,4-\left(\mathrm{OCH}_{2} \mathrm{O}\right) \mathrm{C}_{6} \mathrm{H}_{3}$ & 5 & 81 & $168 \sim 170$ & -- \\
\hline
\end{tabular}

*Yields of the isolated products.

In conclusion, an efficient, simple procedure for the synthesis of 3-aryl-2-mercaptoacrylic acid derivatives from the corresponding the hydrolysis of 5-(benzylidene)rhodanines in alkaline solution under microwave irradiation has been developed.

\section{Experimental Section}

General Procedures. All compounds prepared were characterized by ${ }^{1} \mathrm{H}$ NMR, IR and element analyses and were described in the experimental section. Melting points were determined in XT-5 digital melting point instrument and uncorrected. IR spectra were recorded on a Nicolet Avatar 360 FT-IR spectrometer. ${ }^{1} \mathrm{H}$ NMR spectra were measured at a Burke $400 \mathrm{MHz}$ NMR spectrometer using TMS as internal standard and DMSO- $\mathrm{d}_{6}$ as solvent. MS spectra were obtained on a LCQ Advanbage instrument. Elemental analyses were determined using Perkin-Elmer 240C Elemental Analysis. The reactions were carried out with a modified commercial microwave oven (Sanle WP650D 650w) under atmospheric pressure. 5-(benzylidene)rhodanines were prepared according to the literature. ${ }^{11}$ Other chemicals were available commercially.

General experimental procedure. A mixture of the 5-(benzylidene)rhodanines (2mmol), $15 \%$ sodium hydroxide $(10 \mathrm{~mL})$ in an Erlenmeyer flask $(25 \mathrm{ml})$ equipped with reflux condenser were irradiated in a microwave oven for 3 6 minutes (as indicated by TLC). The mixture was then cooled to room temperature. The concentrated hydrochloride acid was added. The precipitate formed was filtered out and recystallized from ethanol. 
4a. M.p. 135 136 ${ }^{\circ} ;{ }^{1} \mathrm{H}$ NMR (400 MHz, DMSO-d6) $\delta$ 7.95(s, 1H, CH=), 7.43(s, 5H, Ar-H), 4.67 (s, 1H, SH); IR (KBr, cm ${ }^{-1}$ ): 3043, 2566, 1665, 1593, 1505, 1447, 1180, 801; LC/MS (ESI): m/e = 179(M-H) ${ }^{-}$; Anal. Calcd. for $\mathrm{C}_{9} \mathrm{H}_{8} \mathrm{O}_{2} \mathrm{~S}$ : C 59.98; H 4.47; Found: C 60.12; H 4.35.

4b. M.p. $140 \sim 142^{\circ}$; ${ }^{1} \mathrm{H}$ NMR (400 MHz, DMSO-d6) $\delta 7.96(\mathrm{~s}, 1 \mathrm{H}, \mathrm{CH}=), 7.69(\mathrm{~d}, 2 \mathrm{H}, \mathrm{J}=8.8 \mathrm{~Hz}$, Ar-H), $6.98(\mathrm{~d}, 2 \mathrm{H}, \mathrm{J}=8.8 \mathrm{~Hz}, \mathrm{Ar}-\mathrm{H}), 4.65(\mathrm{~s}, 1 \mathrm{H}, \mathrm{SH}), 3.84\left(\mathrm{~s}, 3 \mathrm{H}, \mathrm{CH}_{3} \mathrm{O}\right)$; IR $\left(\mathrm{KBr}, \mathrm{cm}^{-1}\right): 3103$, 2999, 2568, 1655, 1588, 1508, 1439, 1176, 805; LC/MS (ESI): m/e = 209 (M-H) ${ }^{-}$; Anal. Calcd. for $\mathrm{C}_{10} \mathrm{H}_{10} \mathrm{O}_{3} \mathrm{~S}$ : C 57.13; H 4.78; Found: C 57.19; H 4.56.

4c. M.p. 115 116 $6^{\circ}$ ' ${ }^{1} \mathrm{~N}$ NR (400 MHz, DMSO-d6) $\delta 8.23$ (s, 1H, CH=), 7.80 7.42 (m, 3H, furyl-H), 4.69 (s, 1H, SH); IR (KBr, cm $\left.{ }^{-1}\right): 3124,2560,1669,1580,1505,1423,1182,802$; LC/MS (ESI): $\mathrm{m} / \mathrm{e}=169(\mathrm{M}-\mathrm{H})^{-}$; Anal. Calcd. for $\mathrm{C}_{7} \mathrm{H}_{6} \mathrm{O}_{3} \mathrm{~S}: 49.40 ; \mathrm{H}$ 3.55; Found: C 49.52; H3.46.

4d. M.p. 150 152 ; ${ }^{1} \mathrm{H}$ NMR (400 MHz, DMSO-d6) $\delta$ 7.97(d, 1H, 8.2 Hz, CH=), 6.95 7.44(m, 7H, Ar-H, -CH=CH-), 4.89 (s, 1H, SH); IR (KBr, cm $\left.{ }^{-1}\right): 3126,2601,1675,1610,1560,1425$, 1180, 805; LC/MS (ESI): m/e = $205(\mathrm{M}-\mathrm{H})^{-}$; Anal. Calcd. for $\mathrm{C}_{11} \mathrm{H}_{10} \mathrm{O}_{2} \mathrm{~S}$ : C 64.07; H 4.89; Found: C $64.21 \mathrm{H} 4.76$.

4e. M.p.140 142 ${ }^{\circ}{ }^{1} \mathrm{H}$ NMR (400 MHz, DMSO-d6) $\delta 7.91(\mathrm{~s}, 1 \mathrm{H}, \mathrm{CH}=), 8.31(\mathrm{~d}, 2 \mathrm{H}, \mathrm{J}=8.8 \mathrm{~Hz}$, Ar-H), 7.82 (d, 2H, J = 8.8Hz, Ar-H), 4.90(s, 1H, SH); IR (KBr, cm $\left.{ }^{-1}\right): 3113,2560,1678,1592$, 1517, 1424, 1178, 800; LC/MS (ESI): m/e = $224(\mathrm{M}-\mathrm{H})^{-}$; Anal. Calcd. for $\mathrm{C}_{9} \mathrm{H}_{7} \mathrm{NO}_{4} \mathrm{~S}: \mathrm{C} 47.80 ; \mathrm{H}$ 3.13; Found: C 47.90; H 3.05.

4f. M.p. 179 180 ${ }^{\circ}{ }^{1} \mathrm{H}$ NMR (400 MHz, DMSO-d6) $\delta 7.91(\mathrm{~s}, 1 \mathrm{H}, \mathrm{CH}=), 7.78(\mathrm{~d}, 2 \mathrm{H}, \mathrm{J}=8.0 \mathrm{~Hz}$, Ar-H), 7.12 (d, 2H, J = 8.0Hz, Ar-H), 4.65 (s, 1H, SH), 2.36(s, 3H, $\left.\mathrm{CH}_{3}\right)$; IR $\left(\mathrm{KBr}, \mathrm{cm}^{-1}\right): 3088$, 2919, 2566, 1670, 1592, 1505, 1418, 1185, 801; LC/MS (ESI): m/e = 193(M-H) ${ }^{-}$; Anal. Calcd. for $\mathrm{C}_{10} \mathrm{H}_{10} \mathrm{O}_{2} \mathrm{~S}$ : C 61.83; H 5.19; Found: C 61.98; H 5.07

4g. M.p. 140 143; ${ }^{1} \mathrm{H}$ NMR (400 MHz, DMSO-d6) $\delta 7.87$ (s, 1H, CH=), 7.75 7.43(m, 4H, $\mathrm{Ar}-\mathrm{H}), 4.73$ (s, 1H, SH); IR( $\left.\mathrm{KBr}, \mathrm{cm}^{-1}\right): 3058,2561,1678,1589,1508,1430,1182,802$; LC/MS(ESI): m/e = $213(\mathrm{M}-\mathrm{H})^{-}$; Anal. Calcd. for $\mathrm{C}_{9} \mathrm{H}_{7} \mathrm{ClO}_{2} \mathrm{~S}$ : C 55.95; H 3.29; Found: C56.09; H 3.21 .

4h. M.p. 161 162 ${ }^{\circ}$; ${ }^{1} \mathrm{H}$ NMR (400 MHz, DMSO-d6) $\delta 7.82(\mathrm{~s}, 1 \mathrm{H}, \mathrm{CH}=), 7.62(\mathrm{~d}, 2 \mathrm{H}, \mathrm{J}=8.4 \mathrm{~Hz}$, Ar-H), 7.43 (d, 2H, J = 8.4Hz, Ar-H ), 4.73 (s, 1H, SH); IR $\left(\mathrm{KBr}, \mathrm{cm}^{-1}\right): 3103,2572,1664,1586$, $1505,1422,1180,802^{\circ} \mathrm{LC} / \mathrm{MS}(\mathrm{ESI}): \mathrm{m} / \mathrm{e}=213(\mathrm{M}-\mathrm{H})^{-}$; Anal. Calcd. for $\mathrm{C}_{9} \mathrm{H}_{7} \mathrm{ClO}_{2} \mathrm{~S}$ : C 55.95; $\mathrm{H}$ 3.29; Found: C 56.08; H 3.22.

4i. M.p. $163 \sim 164^{\circ 1} \mathrm{H}$ NMR (400 MHz, DMSO-d6) $\delta 7.99$ (s, 1H, CH=), 7.81(d, 2H, J = 8.8Hz, Ar-H), 7.52(d, 2H, J = 8.8Hz, Ar-H), $4.73(\mathrm{~s}, 1 \mathrm{H}, \mathrm{SH}), 3.15\left(\mathrm{~s}, 6 \mathrm{H}, \mathrm{N}\left(\mathrm{CH}_{3}\right)_{2}\right)$; IR $\left(\mathrm{KBr}, \mathrm{cm}^{-1}\right)$ : 3124, 2575, 1695, 1513, 1436, 1182, 805; LC/MS(ESI): m/e = $222(\mathrm{M}-\mathrm{H})^{-}$; Anal. Calcd. for $\mathrm{C}_{11} \mathrm{H}_{13} \mathrm{NO}_{2} \mathrm{~S}$ : C 59.17; H 5.89; Found: C 59.29; H 5.74.

4j. M.p. 168 170 ; ${ }^{1} \mathrm{H}$ NMR (400 MHz, DMSO-d6) $\delta 7.81(\mathrm{~s}, 1 \mathrm{H}, \mathrm{CH}=)$, 7. 52-7.27 (m, 3H, $\mathrm{Ar}-\mathrm{H}), 4.77$ (s, 1H, SH), 5.99 (s, 2H, $\left.\mathrm{OCH}_{2} \mathrm{O}\right)$; IR (KBr, cm $\left.{ }^{-1}\right): 3083,2905,2821,2576,1654$, 1579, 1500, 1449, 1180, 801; LC/MS (ESI): m/e = $223(\mathrm{M}-\mathrm{H})^{-}$; Anal. Calcd. for $\mathrm{C}_{10} \mathrm{H}_{8} \mathrm{O}_{4} \mathrm{~S}: \mathrm{C}$ 53.56; H 3.60;. Found: C 53.68; H 3.52. 


\section{Acknowledgements}

We thank the National Natural Science Foundation of China (No. 20571029) and Jiangsu Key Laboratory for Chemistry of Low-Dimensional Materials for financial support.

\section{References}

1. Izquierdo, A.; Carrasco, J. Analyst 1984, 109, 605.

2. Izquierdo, A.; Beltrán, J. L. Mikrochimica Acta 1989, 98(1-3), 91.

3. Castineiras, A.; Gil, M. J.; Sevillano, P. J. Molecular Structure 2000, 522, 193.

4. Harhash, A. H.; Elnagdi, M. H.; Abdallah, S. O. Indian J. Chem. 1973, 11, 128.

5. Campaigne, E.; Cline. R. E. J. Org. Chem. 1956, 21, 32.

6. Shi H.; Wang, Z.; Shi, H. Huaxue Shiji 1995, 17, 329; Chem. Abstr., 1996, 124, 316658.

7. Caddick, S. Tetrahedron 1995, 51, 10403.

8. Galema, S. A. Chem. Soc. Rev. 1997, 26, 233.

9. El Ashry, El Sayed H.; Ahmed, A. K. Arkivoc 2006, (ix),1.

10. Kappe, C. O. Angew. Chem. Inter. Ed. Engl. 2004, 43, 6250.

11. Zhou, J-F.; Zhu, F-X.; Song Y-Z.; Zhu, Y-L. Arkivoc 2006, (xiv), 175. 